\title{
RECOVERY OF SHEAR-MODIFIED POLYBUTADIENE SOLUTIONS
}

\author{
C. M. ROLAND* \\ Naval Research Laboratory, Chemistry Division, Code 6120, Washington DC 20375-5342 \\ C. G. ROBERTSON $\dagger$ \\ Bridgestone Americas Center for Research And TeCHNOlogy \\ 1200 Firestone Parkway, AKron, OH 44317-0001
}

\begin{abstract}
We have investigated the recovery of the overshoot in the transient viscosity, the first normal stress coefficient, and the dynamic modulus for entangled polybutadiene solutions subjected to nonlinear shear flow. The molecular-weight dependences of the various time scales (linear viscoelastic relaxation time, entanglement recovery time, and timescale for decay of stress following cessation of shearing) are all consistent with the usual 3.4 power law. Nevertheless, the time for recovery of the stress overshoot and plateau value of the dynamic modulus were substantially longer (by as much as two orders of magnitude) than the linear viscoelastic relaxation time calculated from the Newtonian viscosity and the equilibrium recoverable compliance. These results indicate that complete entanglement recovery requires cooperative chain motions over a length scale exceeding that associated with linear relaxation. This persistence of a disentangled state means that a state of low viscosity and reduced elasticity is retained for an extended time, suggesting that shear modification can be used to facilitate the processing of polymers.
\end{abstract}

\section{INTRODUCTION}

The shear-rate dependence of the viscosity is one of the distinguishing features of the rheology of entangled polymers. The shear thinning is ascribed to a loss of entanglements, resulting from the flow-induced orientation of the chains. ${ }^{1-7}$ Although this loss is reversible, the time scale for entanglement recovery can be very slow. This allows advantage to be taken of the "shearmodified" state during polymer processing; for example, elastic effects such as die swell are much weaker for some time after the shearing. ${ }^{8-15}$ Freeze-drying of dilute polymer solutions has also been investigated as a route to achieving disentangled high-molecular weight polymers, ${ }^{16}$ although at least for polystyrene the results are disappointing. ${ }^{17-19}$

Among the various signatures of a reduced entanglement density, the magnitude of the overshoot in the transient shear stress is especially useful for laboratory studies. At sufficiently high shear rates, the stress passes through a maximum, prior to attaining a steady-state value that reflects the equilibrium degree of entanglement. If the shearing is stopped and immediately resumed, the stress overshoot is weaker or even absent. ${ }^{20}$ Annealing in a quiescent state allows the entanglements to reform via Brownian motion, so that upon resumption of the shearing the stress overshoot returns, with a magnitude dependent on the duration of the annealing. ${ }^{21-25}$ Complete recovery of the overshoot (that is, complete re-entanglement) has been reported to require substantially longer times than the time for stress relaxation after cessation of the shearing. ${ }^{21,23}$ Parenthetically, we note that stress overshoots are also observed for unentangled polymers, with similar shear-modification effects; however, these are due simply to orientation, rather than disentanglement, and the overshoot magnitudes are much weaker. ${ }^{26,27}$

Recently we determined the variation with molecular weight, $\mathrm{M}$, of the time for recovery of the stress overshoot, $\tau_{\mathrm{R}, \eta}$, in interrupted shear flow experiments on entangled 1,4-polybutadiene solutions. ${ }^{28}$ In accord with the molecular weight dependence of the linear viscoelastic properties, we found that the recovery kinetics are consistent with a 3.4 power dependence on M. In the present work, we analyze the recovery of the elasticity (from the first normal force data) and the

* email: roland@nrl.navy.mil

$\dagger$ email: RobertsonChristopher@bfusa.com 
dynamic shear modulus in the rubbery plateau regime. The former reflects chain orientation and the latter is due to the transient entanglement network; thus, both provide a measure of the reentanglement process. We have also carried out some preliminary nonlinear creep experiments to verify the absence of inhomogeneous flow, as has been reported recently for well-entangled polymer solutions sheared at very large stresses. ${ }^{29,30}$

\section{EXPERIMENTAL}

The 1,4-polybutadienes, anionically polymerized using butyl lithium as an initiator, are listed in Table I. They had $\sim 9 \%$ vinyl repeat units and low polydispersity ( $\leq 1.05)$. Details of their synthesis can be found in ref. 28. Solutions in an aromatic oil (Sundex 790 from Sun Oil Co.) were prepared by co-dissolution in hexane, with the hexane subsequently removed by evaporation under vacuum. For the startup transient experiments, the polymer volume fraction was $\varphi=$ 0.52 . The entanglement molecular weight, $\mathrm{M}_{\mathrm{e}}=1.85 \mathrm{~kg} / \mathrm{mol}$ for neat $\mathrm{PB}^{31}$, varies as $\varphi^{-1}$ for these solutions. ${ }^{28}$ The calculated number of entanglements per chain is listed in Table I for each sample. Some experiments were also performed on a more dilute solution $(\varphi=0.15)$ of the highest molecular weight PB.

TABLE I

Relaxation And Recovery Times for 1,4-Polybutadiene Solutions $\left(\varphi=0.52 ; \mathrm{T}=25^{\circ} \mathrm{C}\right)$

\begin{tabular}{lcccccccc}
\hline Polymer & $\begin{array}{c}\mathrm{M}_{\mathrm{w}} \\
(\mathrm{kg} / \mathrm{mol})\end{array}$ & $\mathrm{M}_{\mathrm{w}} \varphi / \mathrm{M}_{\mathrm{e}}{ }^{a)}$ & $\begin{array}{c}\log \eta_{0} \\
(\mathrm{~Pa} \mathrm{~s})\end{array}$ & $\begin{array}{c}\dot{\gamma} \\
\left(\mathrm{s}^{-1}\right)\end{array}$ & $\begin{array}{c}\log \tau_{0} \\
(\mathrm{~s})\end{array}$ & $\begin{array}{c}\log \tau_{\mathrm{R}, \eta} \\
(\mathrm{s})\end{array}$ & $\begin{array}{c}\log \tau_{\mathrm{R}, \mathrm{G}} \\
(\mathrm{s})\end{array}$ & $\begin{array}{c}\log \tau_{\mathrm{R}, \varphi} \\
(\mathrm{s})\end{array}$ \\
\hline PB61k & 61.0 & 17 & $3.16 \pm$ & 70 & $-1.38 \pm$ & $0.29 \pm$ & --- & $-0.05 \pm$ \\
& & & 0.01 & & 0.02 & 0.03 & & 0.11 \\
\hline PB90k & 89.6 & 25 & $3.70 \pm$ & 20 & $-0.84 \pm$ & $0.59 \pm$ & -- & $0.31 \pm$ \\
& & & 0.01 & & 0.03 & 0.02 & & 0.32 \\
\hline PB107k & 107.4 & 30 & $4.08 \pm$ & 10 & $-0.46 \pm$ & $0.84 \pm$ & $1.59 \pm$ & $0.53 \pm 1$. \\
& & & 0.01 & & 0.03 & 0.02 & 0.05 & \\
\hline PB167k & 166.9 & 47 & $4.62 \pm$ & 2.5 & $0.08 \pm$ & $1.68 \pm$ & $1.96 \pm$ & $0.93 \pm$ \\
& & & 0.02 & & 0.03 & 0.02 & 0.05 & 0.54 \\
\hline
\end{tabular}

a) Using $\mathrm{M}_{\mathrm{e}}(=1.85 \mathrm{~kg} / \mathrm{mol})$ of neat $\mathrm{PB}$.

Controlled shear rate and small-strain oscillatory shear measurements employed a TA Instruments ARES rheometer, using a cone and plate geometry with a diameter equal to $15 \mathrm{~mm}$ and cone angle $=0.1$ radian. Controlled stress experiments were conducted using a TA Instruments AR1000 with a cone and plate geometry (40 $\mathrm{mm}$ diameter; 0.04 radian cone angle). All rheological measurements were done at $25^{\circ} \mathrm{C}$.

\section{RESULTS}

\section{LINEAR VISCOELASTIC CHARACTERIZATION}

A (weight average) terminal relaxation time $\tau_{0}$ can be determined from linear measurements as

$$
\tau_{0}=\eta_{0} J_{S}^{0}
$$


where $\eta_{0}$ is the Newtonian viscosity and $J_{S}^{0}$ the steady-state recoverable compliance. (Note that other definitions of $\tau_{0}$ yield values that differ by a small numerical constant.) ${ }^{32}$ The crossover from Newtonian flow to shear thinning behavior occurs at a shear rate given by ${ }^{33}$

$$
\dot{\gamma}_{c} \cong 0.6 / \tau_{0}
$$

This is the approximate value of the minimum shear rate necessary to ensure disentanglement by flow.

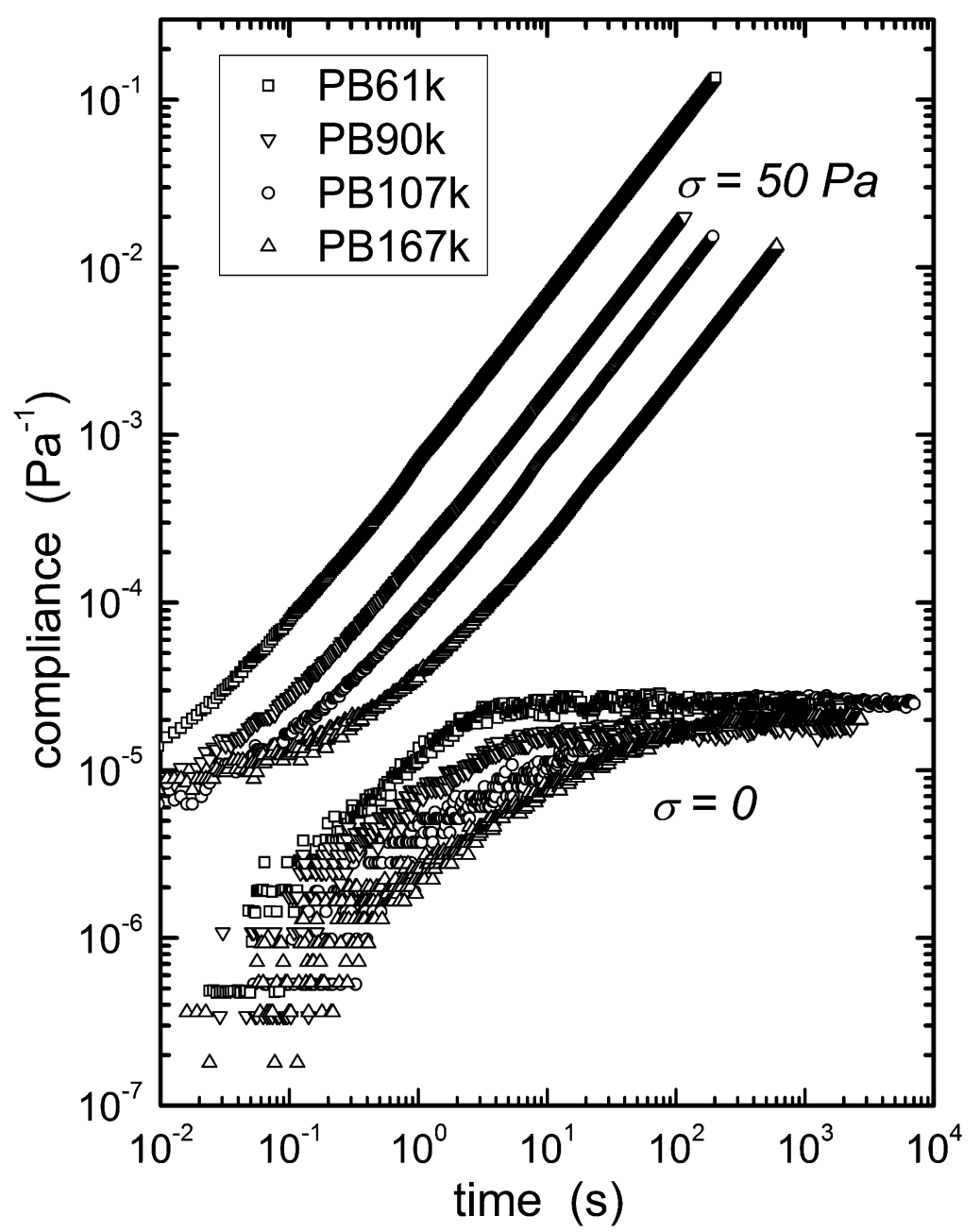

FIG. 1. - Shear compliance measured for the PB solutions at $25^{\circ} \mathrm{C}$. The creep was carried out at $50 \mathrm{~Pa}$ stress, and continued until steady state was attained. The ensuing stress-free recovery reaches a plateau at long time that is independent of molecular weight, $J_{s}^{0}=21 \pm 3 \mathrm{MPa}^{-1}$.

The terminal viscosities measured in shear flow ${ }^{28}$ are listed in Table I. The recoverable compliance was determined by two methods. From linear creep experiments, $J_{S}^{0}$ can be obtained directly from the recovery from steady state creep flow. These measurements are shown in Figure 1. The equilibrium recoverable compliance is the same within experimental error for all samples, 
$J_{S}^{0}=21 \pm 3 \mathrm{MPa}^{-1}$. Note also that the creep recovery levels off to a plateau at longer times, indicating the absence of a high molecular weight tail in the distribution (which may be very difficult to detect analytically.) ${ }^{34,35} \mathrm{~A}$ second measure of $J_{S}^{0}$ is from Newtonian shear flow data using

$$
J_{s}^{0}=\frac{\Psi_{0}}{2 \eta_{0}^{2}}
$$

where $\Psi_{0}$ is the first normal stress coefficient (the ratio of the first normal stress difference to the square of the shear rate, measured at low shear rate). Representative results are shown in Figure 2 for PB107k; for all samples, we obtain $J_{S}^{0}=19 \pm 1 \mathrm{MPa}^{-1}$, equal within the error to the value determined directly by creep recovery.

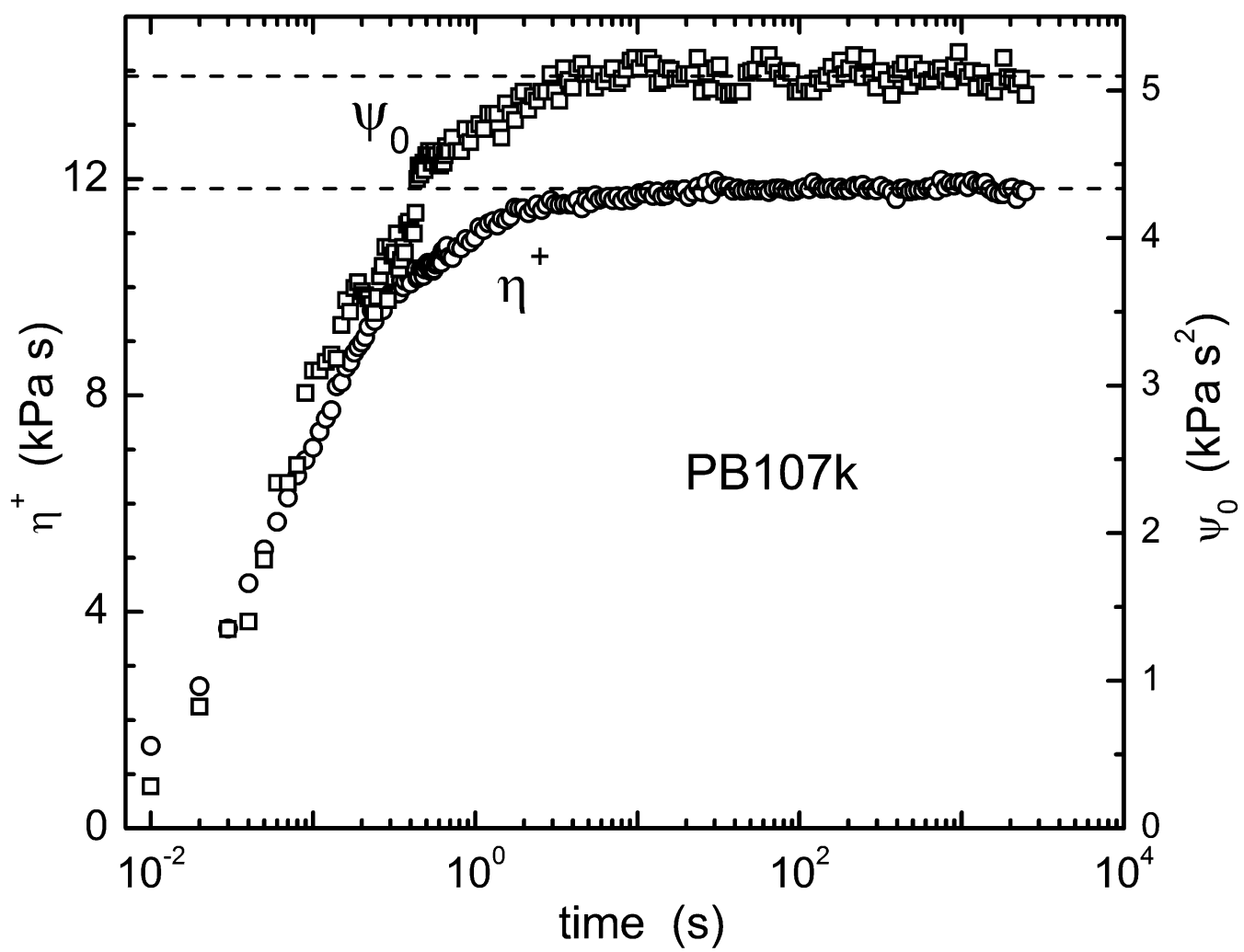

FIG. 2. - Transient viscosity ( $\bigcirc)$ and first normal stress coefficient ( $\square$ ) for the PB107k solution at $25^{\circ} \mathrm{C}$.

The steady-state values, indicated by the horizontal dashed lines, were used to calculate the equilibrium recoverable compliance (Equation 3), yielding a mean value of $19 \pm 1 \mathrm{MPa}^{-1}$ for the four solutions.

This is consistent with the $J_{s}^{0}$ determined from creep recovery (Figure 1).

From Equation 1 we calculate $\tau_{0}$, and the minimum $\dot{\gamma}$ for flow-induced disentanglement is estimated from Equation 2. For the nonlinear shear flow experiments, we choose a value of $\dot{\gamma}=$ $2 / \tau_{0}$, which is 3.3 times $\dot{\gamma}_{c}$; these shear rates are listed in Table I. The highest shear rate which could be employed is governed by the need to avoid edge fracture. Experiments on each solution were carried out to verify that the sample remained intact and homogeneous during shearing at the selected rates. Although edge fracture could be observed after sufficiently long shearing times 
(corresponding to shear strains $>50$ ), the duration of the shear flow in the experiments discussed below was more than a factor of two shorter. In Figure 3 are displayed master curves of the dynamic storage modulus and the dynamic viscosity for the two highest molecular weight PB solutions. The frequency, $\omega$, corresponding to the shear rate used in the nonlinear experiments is indicated. Note that it is beyond the Newtonian plateau.

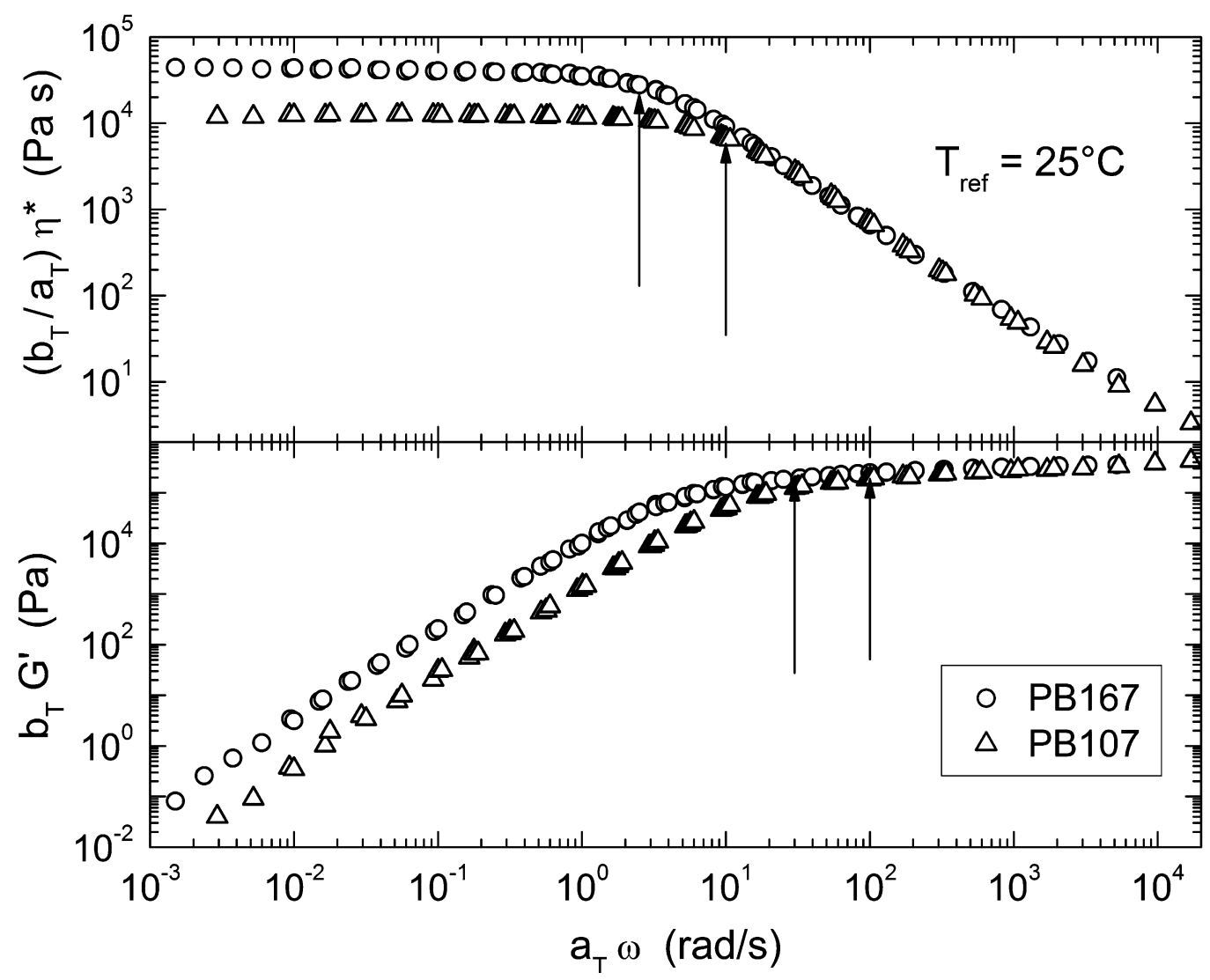

FIG. 3. - (Upper panel) Master curves of the dynamic viscosity for two PB solutions, with arrows indicating the frequency corresponding to $\dot{\gamma}_{c}$ for the non-linear shear experiments.

(Lower panel) Master curve of the dynamic storage modulus, with the arrows indicating the frequency used to measure the plateau modulus during recovery of the entanglements.

\section{NON-LINEAR SHEAR FLOW}

During shearing at 3.3 times $\dot{\gamma}_{c}$, the PB solutions disentangle, giving rise to a maximum in both the shear and normal stresses. This is illustrated in Figure 4, showing typical data for PB90k. For all solutions, the shear stress overshoot was observed at a shear strain $=2.76 \pm 0.26$, with a peak height $1.32 \pm 0.02$ times larger than the steady state stress. The normal force data are much noisier than the shear stress measurements, but the maximum occurred at a shear strain of about 6 , decreasing weakly with molecular weight. These results agree with those of Menezes and Graessely, ${ }^{24}$ who found the strain for the maximum in $\Psi_{0}$ was $2.3 \pm 0.2$ times greater than the strain for the shear stress maximum. 


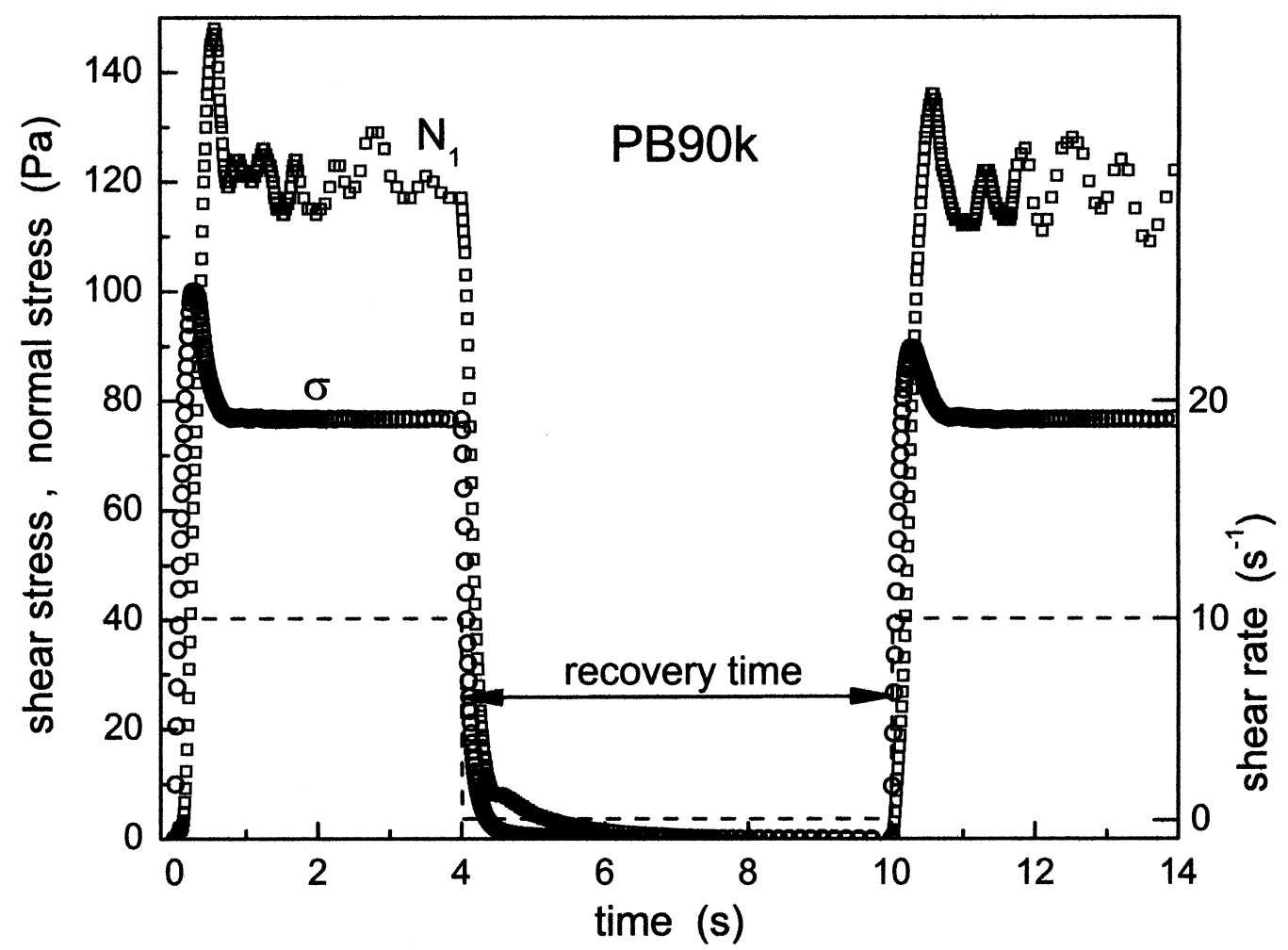

FIG. 4. - Interrupted shear flow experiment (representative data for PB90k solution), showing the overshoot in both the shear stress $\left(=\eta^{+} \dot{\gamma}\right)(\bigcirc)$ and the first normal stress $\left(=\Psi_{0}^{+} \dot{\gamma}^{2}\right)(\square)$. Following a variable time period without flow (as indicated by the arrow), the shearing was resumed with the subsequent stress maxima having a magnitude dependent on the recovery time.

The shear modification experiments, which followed those of Stratton and Butcher, ${ }^{23}$ are depicted in Figure 4. The shear flow was stopped after steady state was reached, and following some time period in a quiescent state, the flow was resumed at the same rate. During the $\dot{\gamma}=0$ period, the stress decays in a complex fashion (Figure 5). Some of the "structure" in these data at short times $(<0.03 \mathrm{~s})$ may reflect instrument problems; in particular, there is some uncertainty in the exact time at which flow had ceased. The normalized decay curves do not superpose when shifted along the time axis; that is, the apparent molecular weight dependence increases with increasing extent of the decay. This is consistent with the idea that the entanglement loss during the prior shearing accelerates the relaxation. ${ }^{7,36}$ Previous studies have also found that the rate of decay depends on the prior shear rate. ${ }^{23,37}$ The long time tail of the relaxation after cessation of the flow ( $>90 \%$ of the decay) does exhibit the expected $\mathrm{M}^{3.4}$ dependence. 


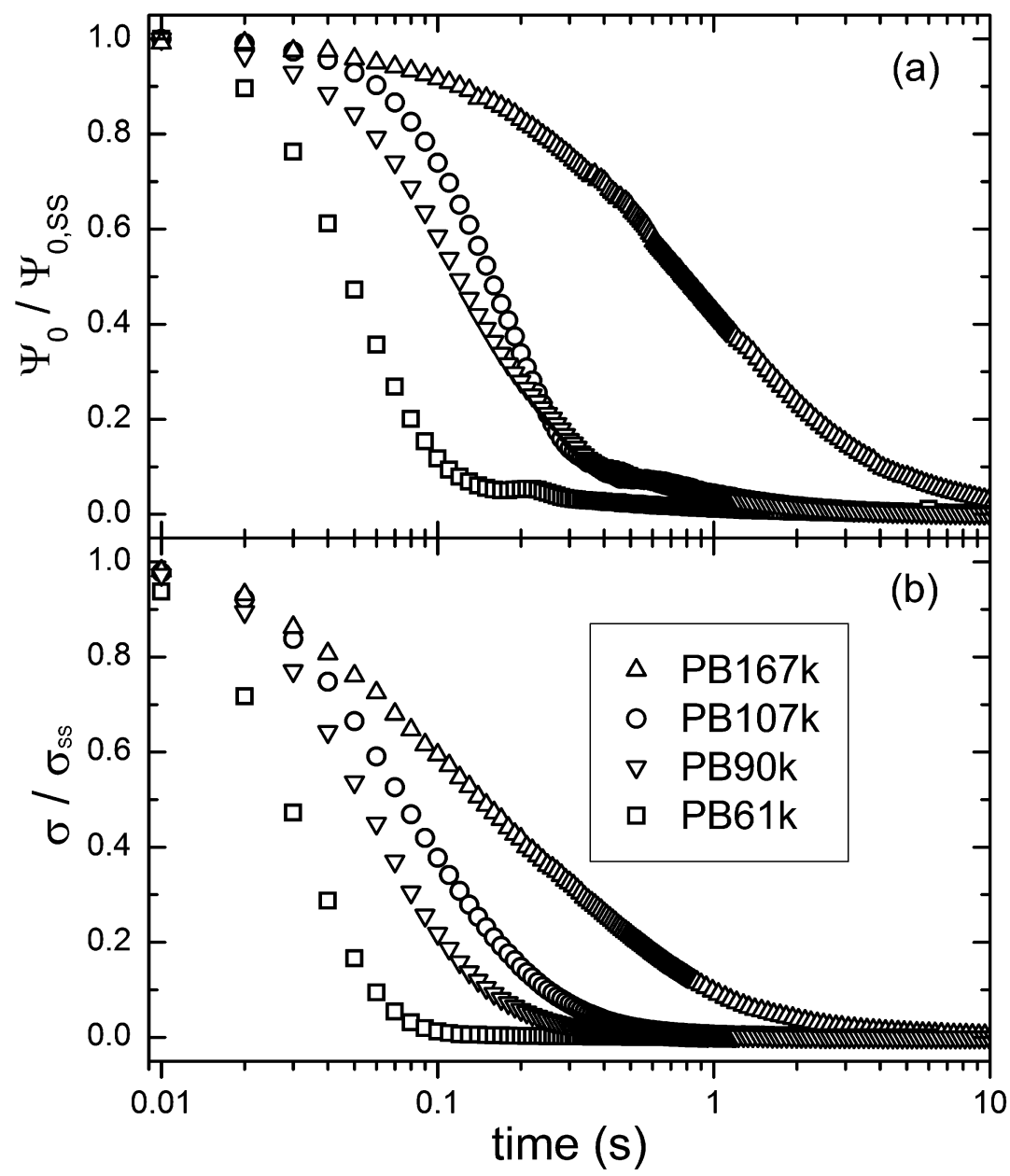

FIG. 5. - Relaxation after cessation of shearing of (a) first normal stress coefficient and (b) shear stress.

As illustrated in Figure 4, the maxima in both the shear viscosity and normal stress coefficient reappeared upon resumption of the shear, with magnitudes dependent on the time at $\dot{\gamma}=0$. The shear stress recovery data, presented previously ${ }^{28}$ and reproduced in Figure 6, are fitted using the exponential function proposed by Stratton and Butcher ${ }^{23}$

$$
\eta_{\max }^{+}(t)=\eta_{s s}+A_{\eta}\left(1-\exp \left[-t / \tau_{R, \eta}\right]\right)
$$

where $\mathrm{A}_{\eta}$ is a constant, $t$ is the time since cessation of the shearing, and $\tau_{R, \eta}$ quantifies the time scale for the recovery of the overshoot in the transient viscosity. A similar analysis of the normal force data using

$$
\Psi_{0}(t)=\Psi_{0, S S}+A_{\Psi}\left(1-\exp \left[-t / \tau_{R, \Psi}\right]\right)
$$

is included in Figure 6, with the characteristic recovery times plotted in the inset. 


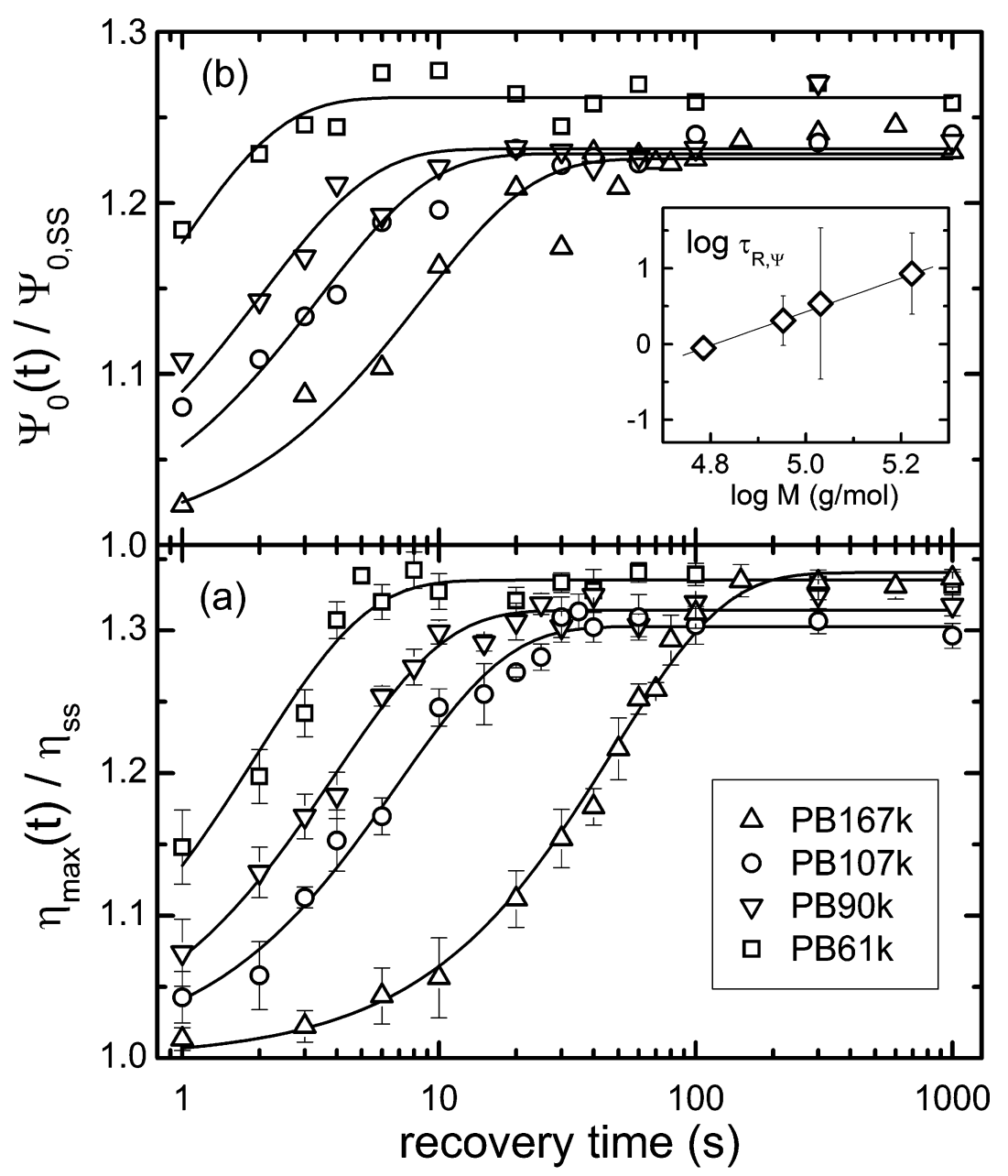

FIG. 6. - (a) Magnitude of overshoot in transient viscosity, normalized by the steady state viscosity, as a function of the rest time between shearing. Data represent the average of three measurements; lines are fits

to Equation 4. (b) Magnitude of overshoot in first normal stress coefficient, normalized by the steady state value of $\Psi_{0}$, as a function of the rest time between shearing. Data represent the average of three measurements; lines are fits to Equation 5. The recovery times for $\Psi_{0}$ (shown in the inset) vary with molecular weight as $\mathrm{M}^{2.3 \pm 1}$.

The plateau in the dynamic storage modulus, $G_{N}^{0}$, is proportional to the entanglement concentration, so that its magnitude can be used to follow the re-entanglement kinetics. Accordingly, we carried out shear flow measurements, using the same conditions as above; however, after the cessation of the flow, a dynamic shear strain $(=0.5 \%)$ was imposed, rather than resuming the steady shearing. The dynamic frequency is indicated in Figure 3. It was an order of magnitude faster than the shear flow rate, which is sufficiently high to fall within the rubbery plateau regime. Figure 7 shows the change in the storage modulus with time after cessation of the shearing. (These experiments were conducted only for the two highest molecular weight samples, because the instrumental lag time ${ }^{38}$ precluded measurements at the short times required for the lower molecular weight samples.) The data were fit to an analog of Equations 4 and 5 


$$
G^{\prime}(t)=G_{\infty}^{\prime}+A_{G}\left(1-\exp \left[-t / \tau_{R, G}\right]\right)
$$

where $\mathrm{G}_{\infty}^{\prime}$ is the equilibrium value $(\mathrm{t} \sim \infty)$ of the storage modulus at the imposed frequency, $\mathrm{A}_{\mathrm{G}}$ is a constant, and $\tau_{\mathrm{R}, \mathrm{G}}$ measures the time for recovery of $G^{\prime}$. The results for the two PB solutions are included in Figure 7.

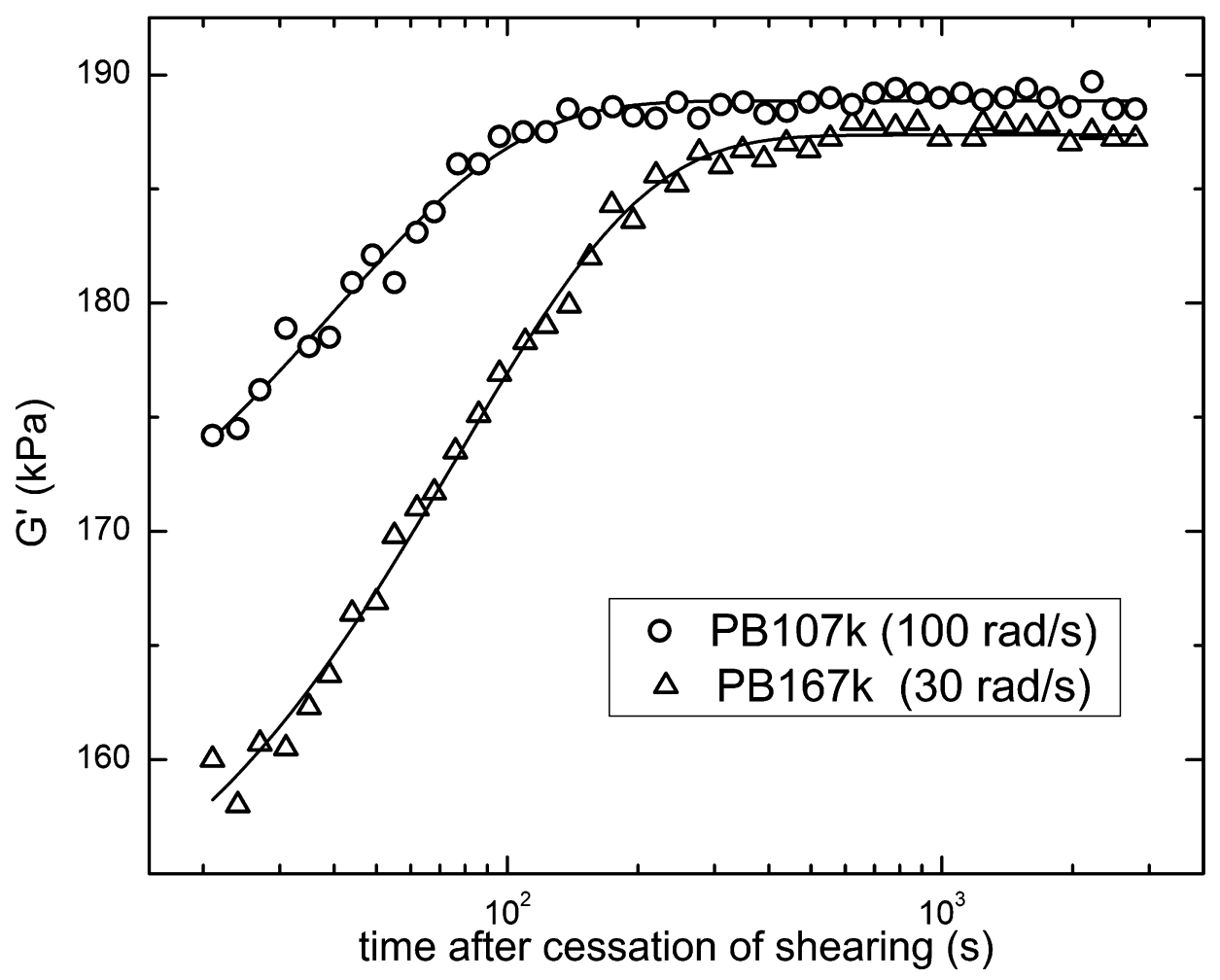

FIG. 7. - Storage modulus measured at the indicated frequencies following cessation of steady state shearing.

\section{HOMOGENEITY OF THE SHEAR FLOW}

Recently, Wang and coworkers have found that entangled polymers will exhibit a yield stress for sufficiently high stresses. ${ }^{29,30}$ As a result, the constitutive relationship between shear stress and shear rate is not continuous and monotonic. This implies that for imposed shear flow such as herein, non-linear rates can result in inhomogeneous flow, whereby the measured stress functions are meaningless. The phenomenon reported by Wang et al. ${ }^{30}$ requires shear stresses that are close in magnitude to the plateau modulus. For the experiments herein, the steady state stress was about $70 \mathrm{kPa}$, which is a factor of 4 to 5 less than the plateau modulus of the solutions (see Figure 3).

Nonlinear creep measurements would more directly assess the presence of yield-like behavior. However, the available instrumentation could not impose high enough stresses. Accordingly, we carried out creep measurements on the PB167k solution after dilution by a factor of 3.5 . Master curves of the dynamic mechanical data are shown in Figure 8. There is a weak plateau in the storage modulus. Using the minimum in the loss tangent as a measure of the plateau modulus, a stress of $5.5 \mathrm{kPa}$ would be the same fraction of the plateau modulus $(\sim 1 / 3)$ as the maxi- 
mum shear stress in the experiments on the more concentrated solutions (Figures 4 - 6). We then measured the creep strain for stresses up to $5.5 \mathrm{kPa}$ (Figure 9). There is no suggestion of any step increase of shear rate, only a smooth increase with increasing magnitude of the stress. This supports the validity of the controlled shear rate experiments on the higher concentration solutions described above. The yield phenomena associated with an entangled-disentangled transition as reported by Wang et al. ${ }^{29,30}$ require higher stresses than used herein.

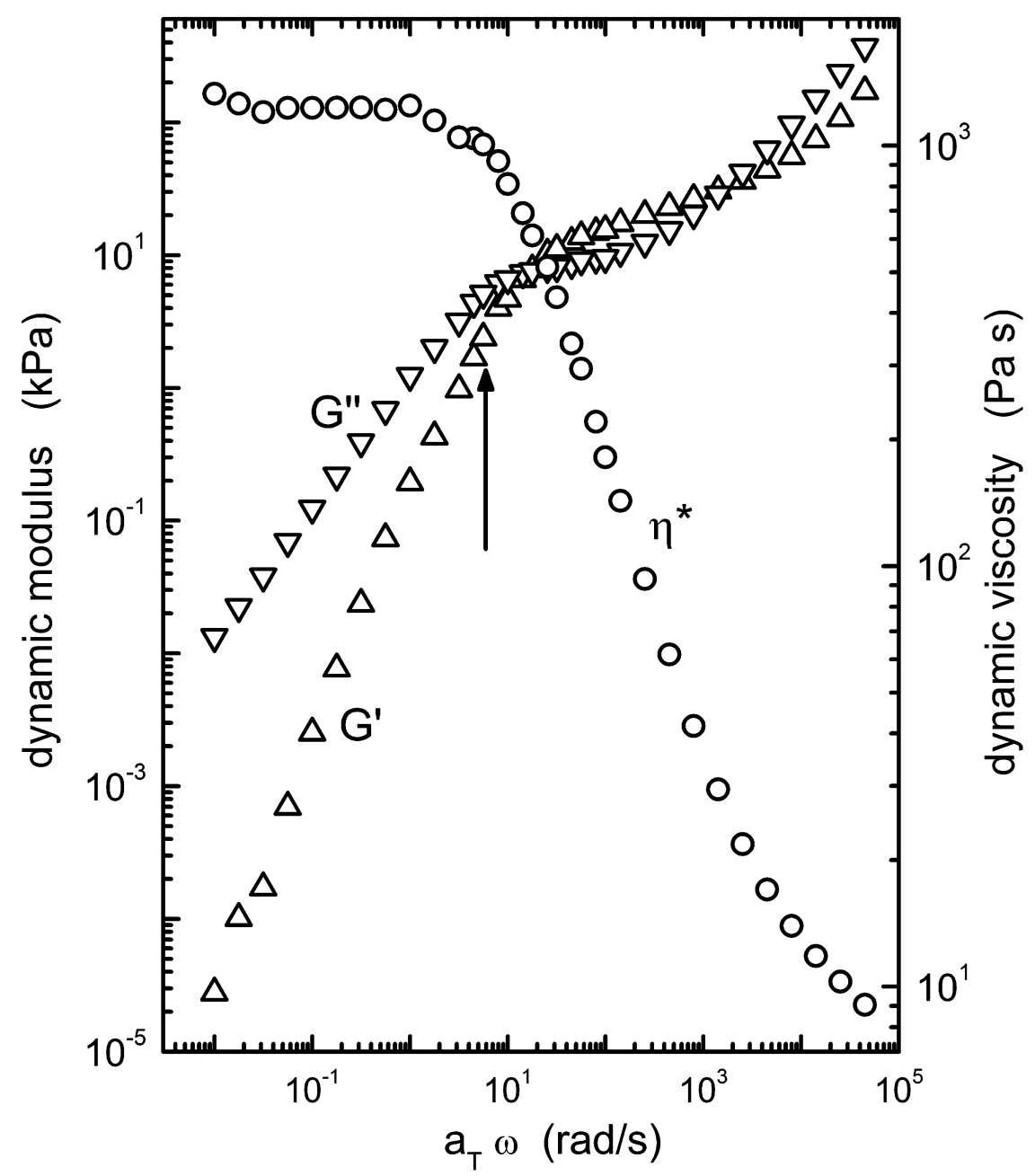

FIG. 8. - Master curves of the dynamic storage $(\triangle)$ and loss modulus $(\nabla)$, and the dynamic viscosity, $=\mathrm{G} * / \mathrm{a}_{\mathrm{T}} \omega(\bigcirc)$, for the diluted PB167 solution. Arrow indicates the frequency corresponding to the rate of the nonlinear creep in Figure 9. 


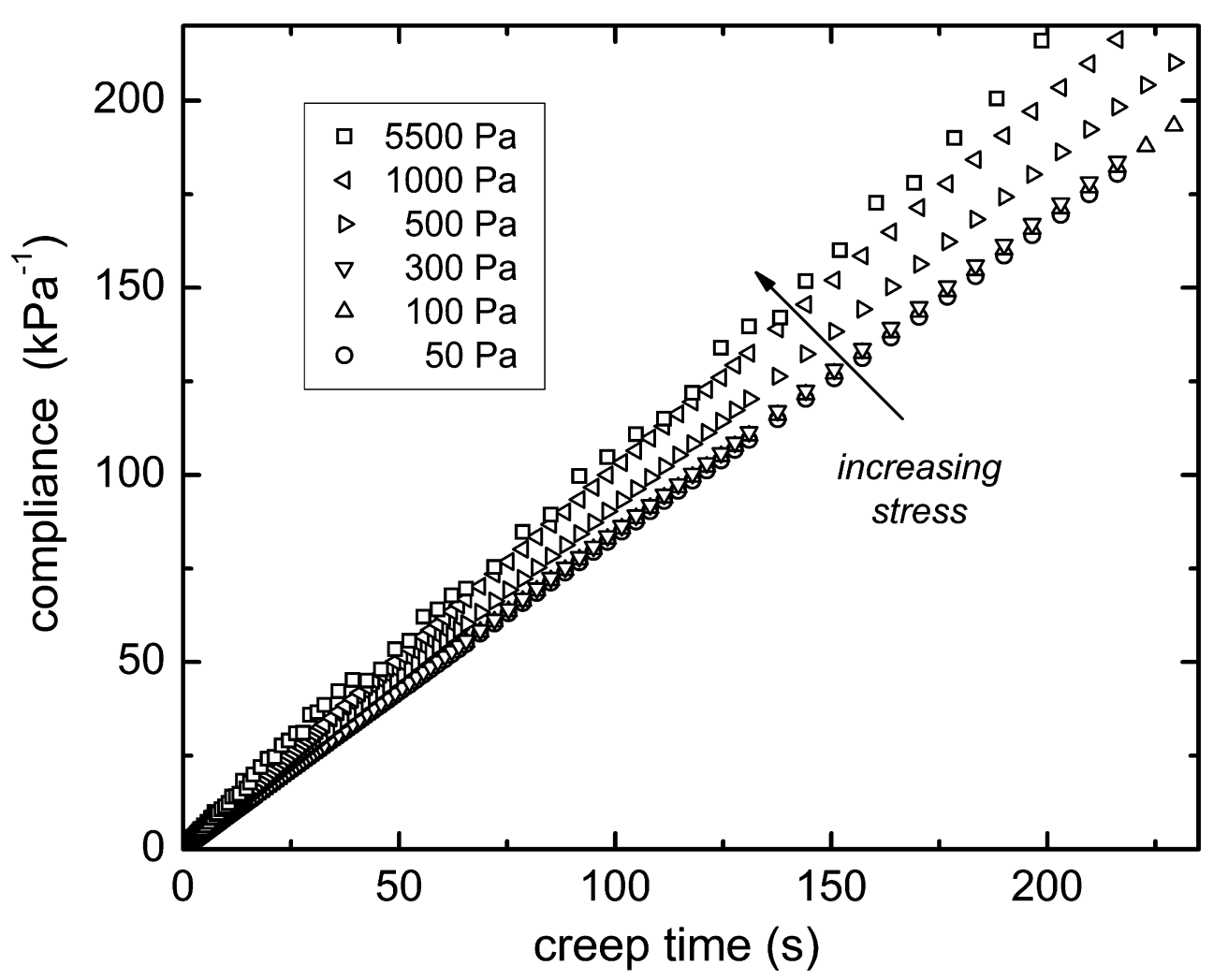

FIG. 9. - Creep compliance for the diluted PB167 solution. Shear rate increases monotonically with magnitude of the shear stress, attaining a value of $5.9 \mathrm{~s}^{-1}$ at $5.5 \mathrm{kPa}$.

\section{DISCUSSION}

Figure 10 shows the terminal relaxation times for the PB solutions obtained from linear viscoelastic measurements (Equation 1). They vary with molecular weight as $\tau_{0} \sim \mathrm{M}^{3.4 \pm 0.2}$, in accord with general expectations ${ }^{39,40}$ and previous experiments on 1,4-polybutadiene. ${ }^{31}$ The recovery times for the $\eta^{+}$overshoot and the storage modulus are included in the figure. Both quantities represent the time required for re-entanglement after non-linear shearing. We find that this process has a molecular weight dependence varying as $M$ to the $3.3 \pm 0.3$ power, which is essentially the same as for $\tau_{0}$. This is interesting because while $\tau_{0}$ describes linear relaxation in the fully-entangled state, $\tau_{\mathrm{R}}$ reflects the dynamics of partially disentangled chains. Notwithstanding their equivalent variation with $\mathrm{M}$, the re-entanglement time scale is substantially longer than the linear relaxation time: $\tau_{R, \eta}$ is $\sim 48$ times $\tau_{0}$ and $\tau_{R, \mathrm{G}}$ is a factor of 160 times larger than $\tau_{0}$. The linear relaxation time is a measure of the time for the entangled chain to diffuse a distance roughly equal to its coil size. Although one expects disentangled chains to exhibit faster motion, the recovery time scale involves an approach to the fully entangled state via Brownian motion. This is a stochastic process, evidently requiring motion over length scales significantly greater than the molecular size. 


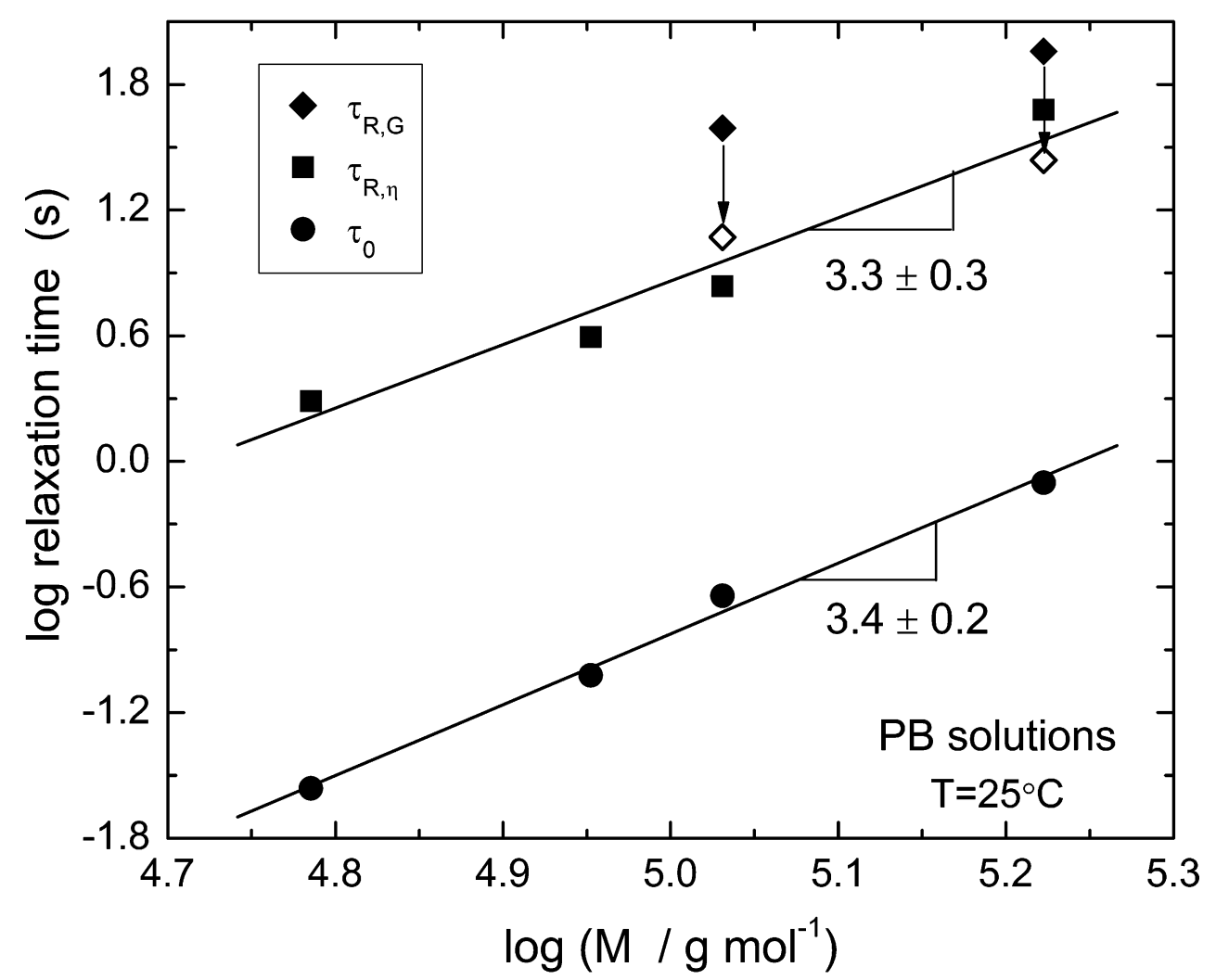

FIG. 10. - Molecular weight dependence of the terminal relaxation time from linear viscoelastic measurements the time for recovery of the overshoot peak in the transient shear stress $(\boldsymbol{\square})$, and the time for recovery of the plateau modulus $(\bullet)$. The latter is also shown after vertically shifting (by a factor of 0.30 ) to superimpose on the $\eta^{+}$data $(\diamond)$; this demonstrates the equivalence of the molecular weight dependences.

Although the direct origin of normal stress is the chain stretching induced by flow, the magnitude of $\Psi_{0}$ is sensitive to entanglements, and thus its recovery should follow the re-entanglement process. The recovery of the normal stress is faster than for the viscosity overshoot (Table 1) although still longer (by about a factor of ten) than $\tau_{0}$. Unfortunately, the scatter in the normal force data precludes an accurate determination of the molecular weight dependence of its recovery kinetics. As shown in the inset to Figure $6 \mathrm{~b}, \tau_{R, \Psi} \propto \mathrm{M}^{2.3 \pm 1}$.

The fact that the recovery time is substantially larger than the linear viscoelastic relaxation time is not predicted by any model, ${ }^{25,41,42}$ but this effect has been observed previously. Startup transient recovery times for polyethylene melts reported by Dealy and Tsang ${ }^{21}$ were a factor of 13 longer than $\tau_{0}{ }^{42}$ Yamaguchi and Gogos ${ }^{8}$ measured the recovery of the plateau modulus for sheared polyethylene melts $(\mathrm{M} \sim 210 \mathrm{~kg} / \mathrm{mol})$. Analysis of their data indicates the time for reentanglement to be a factor of 28 longer than the linear viscoelastic relaxation time. This slow entanglement process is also seen in measurements on a very high molecular weight PB $(\mathrm{M}=$ $\left.1.7 \times 10^{4} \mathrm{~kg} / \mathrm{mol}\right) .{ }^{31}$ The sample, precipitated from solution and molded at low temperature, was found to have a non-equilibrium degree of entanglements after 30 days, although the terminal relaxation time was less than 5 days. Notwithstanding the non-equilibrated nature of the material, viscosity measurements were the basis for a putative confirmation of the $\mathrm{M}^{3}$ dependence of the viscosity (at sufficiently large $\mathrm{M}$ ) predicted by tube models of polymer dynamics. ${ }^{31,40}$

Other workers, studying more dilute but still entangled solutions $(\varphi<0.1)$, have obtained 
$\tau_{R, \eta}$ that were closer to the linear viscoelastic time. ${ }^{23,24,37}$ However, even in these studies, $\tau_{R, \eta}$ was much longer than the time for stress relaxation following cessation of flow.

\section{SUMMARY}

Non-linear shearing was used to reduce the entanglements in concentrated solutions of high molecular weight PB. The recovery of the equilibrium degree of entanglements was followed by measurements of the transient viscosity, normal stress coefficient, and dynamic storage modulus. The kinetics of the re-entanglement are consistent with the 3.4 power dependence on molecular weight found for linear viscoelastic properties. However, the time scale for recovery of the entanglements is much longer (by $c a$. two orders of magnitude) than the linear relaxation time. This can lead to errors in characterizations of the viscoelastic properties of samples whose preparation involved significant deformation or the use of solvent. On the other hand, a reduced entanglement concentration gives more facile flow with weaker elastic effects; thus, shear modification is a route to improved processibility without sacrifice of ultimate physical properties.

\section{ACKNOWLEDGMENTS}

We thank S. Warren of Bridgestone Americas for kindly synthesizing the polybutadienes, and D. J. Plazek and K. L. Ngai for stimulating discussions. The work at NRL was supported by the Office of Naval Research. CGR thanks Bridgestone Americas for permission to publish.

\section{REFERENCES}

${ }^{1}$ G. V. Vinogradov and I. M. Belkin, J. Polym. Sci. A 3, 917 (1965).

${ }^{2}$ J. M. Simmons, J. M. Rheol. Acta 7, 184 (1968).

${ }^{3}$ R. I. Tanner and G. Williams, Rheol. Acta 10, 528 (1971).

${ }^{4}$ H. Batzer, M.H. Wagner and J. Meissner, Makromol. Chem. 181, 1533 (1980).

${ }^{5}$ Y. Isono, N. Obashi and T. Kase, Macromolecules 28, 5145 (1995).

${ }^{6}$ Y. Isono, T. Kamohara, A. Takano and T. Kase, Rheol. Acta 35, 245 (1997).

${ }^{7}$ J. P. Oberhauser, L. G. Leal and D. W. Mead, J. Polym. Sci. Polym. Phys. Ed. 36, 265 (1998).

${ }^{8}$ M. Yamaguchi and C. G. Gogos, Adv. Polym. Testing 20, 261 (2001).

${ }^{9}$ A. Rudin and H.P. Schreiber, Polym. Eng. Sci. 23, 422 (1983).

${ }^{10}$ P. J. R. Leblans and C. Bastiaansen, Macromolecules 22, 3312 (1989).

${ }^{11}$ H. A. Pohl and C. G. Gogos, J. Appl. Polym. Sci. 5, 67 (1961).

${ }^{12}$ M. Rokudai, J. Appl. Polym. Sci. 23, 463 (1979).

${ }^{13}$ H. P. Schreiber, A. Rudin and E. G. Bagley, J. Appl. Polym. Sci. 9, 887 (1965).

${ }^{14}$ A. Ram and L. Izrailov J. Appl. Polym. Sci. 31, 85 (1986).

${ }^{15}$ R. Schertzer, A. Rudin and H. P. Schreiber, J. Appl. Polym. Sci. 31, 809 (1986).

${ }^{16}$ L. P. Chang and H. Morawetz, Macromolecules 20, 428 (1987); ibid. 21, 515 (1988).

${ }^{17}$ A. M. Kotliar, R. Kumar and R. A. Back, J. Polym. Sci., Polym.Phys. Ed. 28, 1033 (1990).

${ }^{18}$ K. J. McGrath, C. M. Roland, and R. G. Weiss, Macromolecules 26, 6127 (1993).

${ }^{19}$ K. J. McGrath and C. M. Roland Macromolecules 25, 1366 (1992).

${ }^{20}$ P. Attane, J. M. Pierrard and G. Turrel, J. Non-Newtonian Fluid Mech. 18, 295 (1985).

${ }^{21}$ J. M. Dealy and W. K.-W. Tsang, J. Appl. Polym. Sci. 26, 1149 (1981).

${ }^{22}$ J. D. Huppler, I. F. MacDonald, E. Ashare, T. W. Spriggs, R. B. Bird and L. A. Holmes, J. Rheol. 11, 181 (1967).

${ }^{23}$ R. A. Stratton and A. F. Butcher, J. Polym. Sci. Polym. Phys. Ed. 11, 1747 (1973).

${ }^{24}$ E. V. Menezes and W. W. Graessley, J. Polym. Sci. Polym. Phys. Ed. 20, 1817 (1982). 
${ }^{25}$ Y. Z. Xu, D. Dekee and C. F. C. M. Fong, J. Appl. Polym. Sci. 55, 779 (1995).

${ }^{26}$ J. D. Moore, S. T. Cui, H. D. Cochran and P. T. Cummings, Phys. Rev. E 60, 6956 (1999).

${ }^{27}$ P. G. Santangelo and C. M. Roland, J. Rheology 45, 583 (2001).

${ }^{28}$ C. G. Robertson, S. Warren, D.J. Plazek and C.M. Roland, Macromolecules 37, 10018 (2004).

${ }^{29}$ P. Tapadia and S. Q. Wang, Phys. Rev. Lett. 91, 198301 (2003).

${ }^{30}$ P. Tapadia and S.Q. Wang, Macromolecules 37, 9083 (2004).

${ }^{31}$ R. H. Colby, L. J. Fetters and W. W. Graessley, Macromolecules 20, 2226 (1987).

${ }^{32}$ C. M. Roland, K. L. Ngai and D. J. Plazek, Macromolecules 37, 7051 (2004).

${ }^{33}$ W. W. Graessley Adv. Polym. Sci. 16, 1 (1974).

${ }^{34}$ H. Mavridis and R. Shroff J. Appl. Polym. Sci. 49, 299 (1993).

${ }^{35}$ S. Pang and A. Rudin Polymer 33, 1949 (1992).

${ }^{36}$ V. Mhetar and L. A. Archer J. Non-Newtonian Fluid Mech. 81, 71 (1999).

${ }^{37}$ J. Sanchez-Reyes and L. A. Archer, J. Rheol. 47, 469 (2003).

${ }^{38}$ When using the ARES and transitioning from steady state to oscillatory shear, the starting position of the steady state motor is set so that steady shearing terminates at the angular position corresponding to the zero position of the dynamic motor. Additionally, a $c a$. 15 second lag is introduced by the instrument between the segments of such a test sequence.

${ }^{39}$ J. D. Ferry, "Viscoelastic Properties of Polymers," $3^{\text {rd }}$ ed. (Wiley, New York, 1980).

${ }^{40}$ M. Doi and S.F. Edwards, "The Theory of Polymer Dynamics" (Clarendon, Oxford, 1986).

${ }^{41}$ M. Takahashi, T. Masuda and S. Onogi, Trans. Soc. Rheol. 21, 337 (1977).

${ }^{42}$ W. K.-W. Tsang and J. M. Dealy, J. Non-Newtonian Fluid Mech. 9, 203 (1981).

[ Paper 50 presented at the Spring Meeting of the Rubber Division, ACS (San Antonio, TX)

May 16-18, 2005, revised February 2006 ] 